\title{
OCLUSIVAS GLOTALES EN EL INGLÉS CRIOLLO LIMONENSE: EVIDENCIAS ACÚSTICAS
}

Mario Portilla

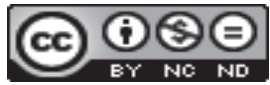

Doi: https://doi.org/10.15517/rfl.v46iExt..43890

URL: https://revistas.ucr.ac.cr/index.php/filyling/index 



\title{
OCLUSIVAS GLOTALES EN EL INGLÉS CRIOLLO LIMONENSE: EVIDENCIAS ACÚSTICAS
}

\author{
GLOTTAL STOPS IN LIMONESE CREOLE ENGLISH: \\ ACOUSTIC EVIDENCE
}

Mario Portilla

\begin{abstract}
RESUMEN
En este artículo se presentan evidencias acústicas de la existencia de oclusivas glotales ante /p, t, k/ en posición final de sílaba y ante vocales al inicio de palabra o precedidas de otra vocal en el inglés criollo limonense, a partir del análisis de diversos oscilogramas y espectrogramas de esta lengua. Palabras clave: oclusivas glotales; inglés criollo limonense; lenguas criollas; fonética acústica; espectrogramas.
\end{abstract}

\begin{abstract}
This paper shows acoustic evidence of glottal stops before /p, t, k/ in syllable-final position and before vowels initially or between them in Limonese Creole English by analyzing some oscillograms and spectrograms of this language.

Keywords: glottal stops; Limonese Creole English; Creole languages; acoustic phonetics; spectrograms.
\end{abstract}

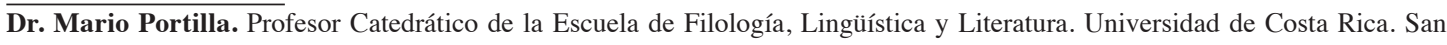
José, Costa Rica.

Correo electrónico: mportillacr@gmail.com 


\section{Antecedentes}

Ninguno de los autores de las descripciones fonológicas del inglés criollo limonense había hecho referencia a la existencia de oclusivas glotales en su nivel fonético antes de la aparición del análisis fonemático de esta lengua realizado por Portilla (1993)¹.

El autor reconoce la intrusión de una oclusiva glotal en posición posvocálica delante de la serie de oclusivas sordas /p, t, k/, especialmente cuando se trata de vocales de cantidad breve.

$$
\begin{aligned}
& \text { /dráp/ } \rightarrow \text { [drá?p] } \quad \text { caer }(<\text { drop }) \\
& / \mathrm{f}^{\prime} \mathrm{t} / \rightarrow\left[\mathrm{f} \mathrm{v}^{\prime} \mathrm{Pt}\right] \quad \operatorname{cortar}(<\mathrm{cut}) \\
& / \mathrm{k}^{\prime} \mathrm{k} / \rightarrow\left[\mathrm{k} \mho^{\prime} \mathrm{Tk}\right] \quad \operatorname{cocinar}(<\operatorname{cook})
\end{aligned}
$$

Esta aparición de la glotal es facultativa cuando las vocales que preceden a las oclusivas sordas son largas.

$$
\begin{aligned}
& / \text { rvop }^{\prime} \rightarrow \text { [ro'o?p] } \sim \text { [ro'op] cuerda }(<\text { rope }) \\
& / \text { tí:t } / \rightarrow\left[\mathrm{tI}^{\prime} \mathrm{i} \mathrm{i} \mathrm{t}\right] \sim\left[\mathrm{t}^{\prime} \mathrm{it}\right] \quad \text { diente }(<\text { teeth }) \\
& / \text { rú:t/ } \rightarrow \text { [róu?t] } \sim\left[\mathrm{tr}^{\prime} \mathrm{it}\right] \quad \text { raíz }(<\text { root }) \\
& / \text { líek } / \rightarrow\left[\mathrm{li}^{\prime} \mathrm{e} \mathrm{e} \mathrm{k}\right] \sim[\text { líek] lago }(<\text { lake })
\end{aligned}
$$

Como se nota en los ejemplos, las vocales largas están constituidas por dos moras. La primera de ellas, en el caso de las vocales medias y altas, es una floja (I, $)$ y la segunda una tensa $(\mathrm{i}, \mathrm{u})^{2}$.

Además, Portilla (1993) señala que, por un lado, la oclusiva glotal se introduce cuando las consonantes $/ \mathrm{p}, \mathrm{t}, \mathrm{k} / \mathrm{se}$ encuentran en posición final de palabra y, por otro lado, que aparece una oclusión glotal en posición inicial de palabra ante cualquier vocal y que esta se intercambia frecuentemente con la fricativa glotal sorda [h].

Por regla general, ninguna vocal puede aparecer fonéticamente en posición inicial absoluta. Así, pues, una vocal que aparezca fonológicamente en dicha posición, debe estar precedida por una constricción glotal, la cual se expresa fonéticamente como una oclusión [?] o como una fricción [h]. (Portilla, 1993, p. 92).

$$
\text { /íet/ } \rightarrow \text { [?íe?t] } \sim \text { [híe?t }] \quad \text { ocho }(<\text { eight })
$$

En resumen, el autor establece la intrusión de una oclusiva glotal después de una vocal, especialmente si es de longitud breve, y ante una consonante de la serie de oclusivas sordas cuando estas se encuentran en posición final (a). Igualmente, esta aparece ante cualquier vocal en posición inicial absoluta (b).

(a) $\quad \varnothing \rightarrow ? / \mathrm{V}-\mathrm{C}_{\mathrm{X}} \# \#, \mathrm{C}_{\mathrm{X}}=/ \mathrm{p}, \mathrm{t}, \mathrm{k} /$
(b) $\quad \varnothing \rightarrow ? / \# \#-\mathrm{V}$

1 Esta lengua también ha sido conocida como mekatelio (mekaytelyuw y otras variantes), inglés criollo de Costa Rica e inglés de Limón, entre otras denominaciones. Otros autores que han realizado descripciones fonológicas de la lengua son Bernard (1969), Wolfe (1970), Wright $(1974,1975)$ y Herzfeld (1978; 2002).

2 El inglés criollo limonense presenta un sistema vocálico de cinco segmentos, con dos series de vocales breves y largas: /I, i:,, , u:, e, Ie, $\Lambda$, ซo, a, a:/ <i, ii, u, uu, e, ie, o, uo, a, aa>. Dado que la diferencia fonética entre una vocal breve y una larga, cuando se trata de vocales medias y altas, se debe solamente a la cualidad de la segunda parte de la mora, la cual es siempre tensa, se considera que los diptongos / Ie, $\mho \mathrm{o} /$ representan los correlatos vocálicos de cantidad larga de las vocales /e, $\Lambda$ respectivamente, de la misma forma que sucede con los segmentos /i:, u:/ [ii, vu] que lo son de /I, $/$. Además, el inglés criollo limonense es una lengua de acento tonal, cuya culminación máxima se expresa por medio de un tono alto sobre la vocal (marcado ortográficamente solo en las palabras polisilábicas por medio de una tilde). 
El objetivo principal de este trabajo es mostrar evidencia empírica que confirme la existencia de oclusivas glotales en el inglés criollo limonense, por medio del análisis acústico de materiales de esta lengua ${ }^{3}$.

También, se pretende describir acústicamente las características fonéticas de su realización, así como confirmar o no las condiciones que propician su aparición.

\section{Caracterización acústica de las oclusivas glotales}

Desde un punto de vista articulatorio, un cierre glotal se describe como una oclusiva sorda pulmónica que se produce con la obstrucción total o parcial de la glotis por medio de las cuerdas vocales y también a veces con la constricción de las cuerdas vocales falsas (Kalita et al. 2017, p. 1). Normalmente, las oclusivas glotales varían a causa de la cualidad de voz del hablante, su posición en la sílaba y la frase en que se hallen.

Desde una perspectiva acústica, una oclusiva glotal sorda aparece en los espectrogramas como la interrupción de las vibraciones de la voz. En algunas lenguas a veces ocurre una laringalización o voz quebrada (creaky voice), especialmente de las vocales, junto con la oclusiva glotal o en lugar de esta.

\section{Realización de las oclusivas glotales ante consonantes oclusivas sordas}

Diversos oscilogramas y espectrogramas muestran la aparición de una oclusiva glotal sorda en este contexto, expresada especialmente por medio una laringalización o voz quebrada. La Figura 1 presenta la realización de la palabra bit $\left[\mathrm{bi}^{\prime}\right.$ ? $\left.\mathrm{t}^{\mathrm{h}}\right]$ 'trozo'.

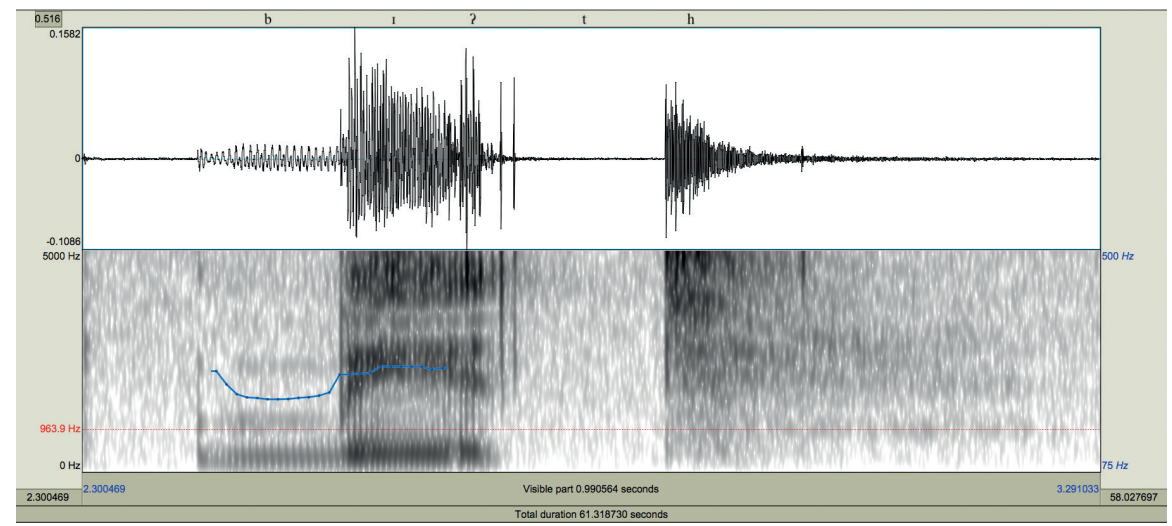

Figura 1. Oscilograma y espectrograma de la palabra bit [bi'?t $\left.{ }^{\mathrm{h}}\right]$ 'trozo'

En esta figura, la palabra bit 'trozo' muestra pulsaciones glotales irregulares y no periódicas al final de la vocal corta /I/ [I], las cuales se notan claramente en la onda del sonido que dibuja el oscilograma en la parte superior. Estas corresponden a la voz quebrada, que es el correlato fonético de la oclusión glotal percibida por Portilla (1993). Luego de la voz quebrada,

3 Los materiales utilizados corresponden a una lista de palabras y frases educidas a la consultora lingüística Marcia Reed Chambers en agosto de 2009 en el laboratorio de fonética del Departamento de lingüística de la Universidad de Costa Rica. Los archivos digitales fueron analizados con el programa Praat (versión 5.2.25). 
hay una ausencia de voz propia de la oclusiva sorda, seguida después de una fricativa glotal, que equivale a la aspiración de dicha consonante.

La Figura 2 recoge un extracto de solamente la vocal de este vocablo. Como se aprecia, el tono, marcado por la línea azul, marca el ámbito de las pulsaciones regulares en la vocal y su límite.

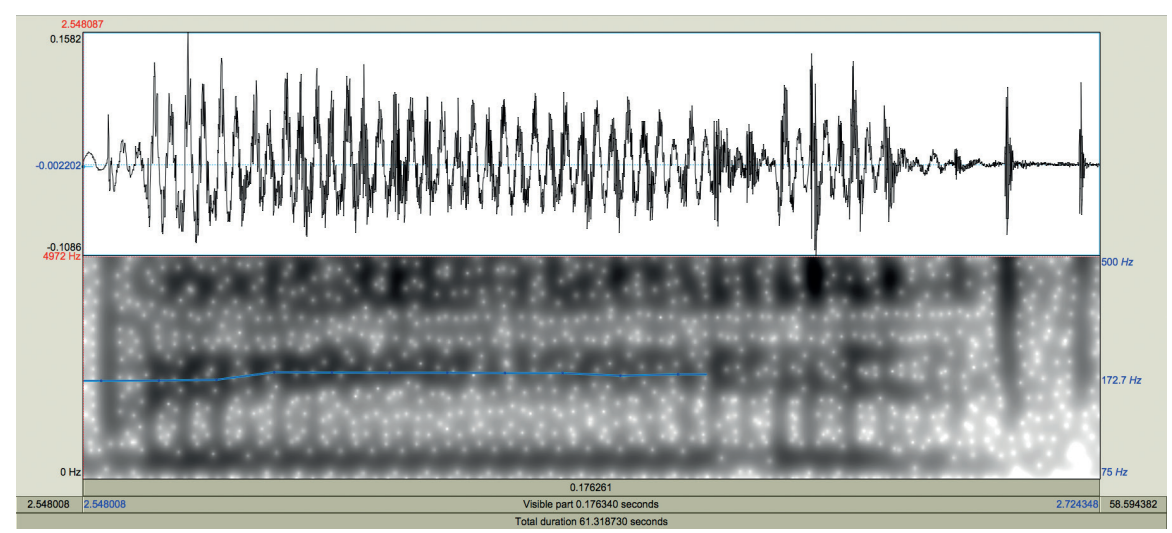

Figura 2. Oscilograma y espectrograma de la vocal [I] en palabra bit 'trozo'

Por su parte, la Figura 3 presenta la realización del vocablo biit [bi' it ${ }^{\text {h] }}$ 'golpear'. En esta vocal no hay indicios de voz quebrada. La onda de sonido de la vocal larga es regular, como se ve claramente en el oscilograma.

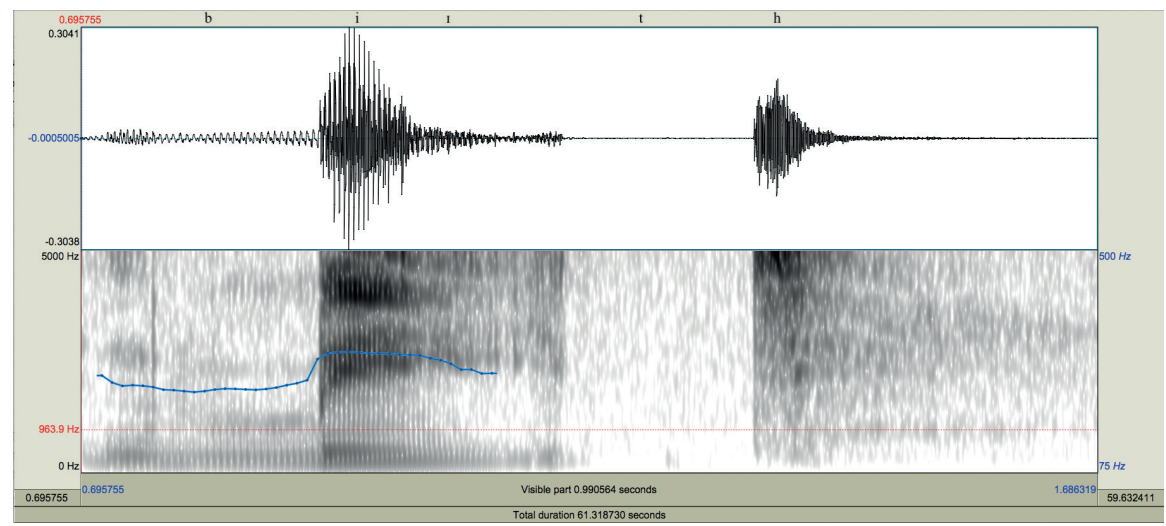

Figura 3. Oscilograma y espectrograma de la palabra biit [br' it ${ }^{\text {hy }}$ 'golpear'

Del mismo modo, en la Figura 4, el extracto de la vocal larga /i:/ [ii] revela que la onda de sonido mantiene estable las pulsaciones glotales en cada una de las moras. Evidentemente, la primera mora [I] presenta longitudes de onda mayores que la segunda [i], como ya ha sido descrito antes por Portilla (2010, p. 180). 


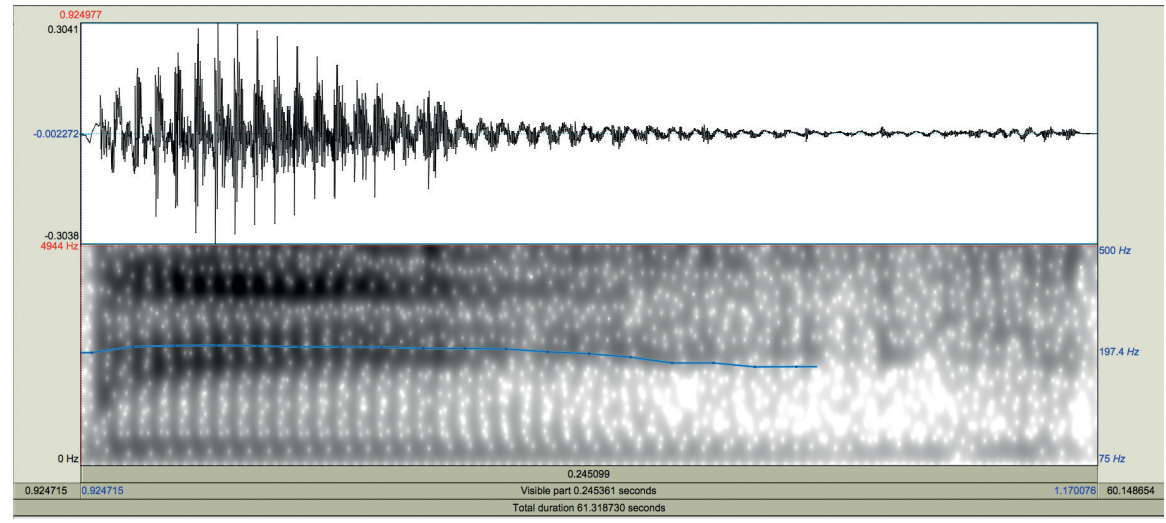

Figura 4. Oscilograma y espectrograma de la vocal [ir] en palabra la palabra biit 'golpear'

Con las consonantes /p/ y /k/ ocurre una situación análoga a la descrita para el segmento /t/ en similares condiciones.

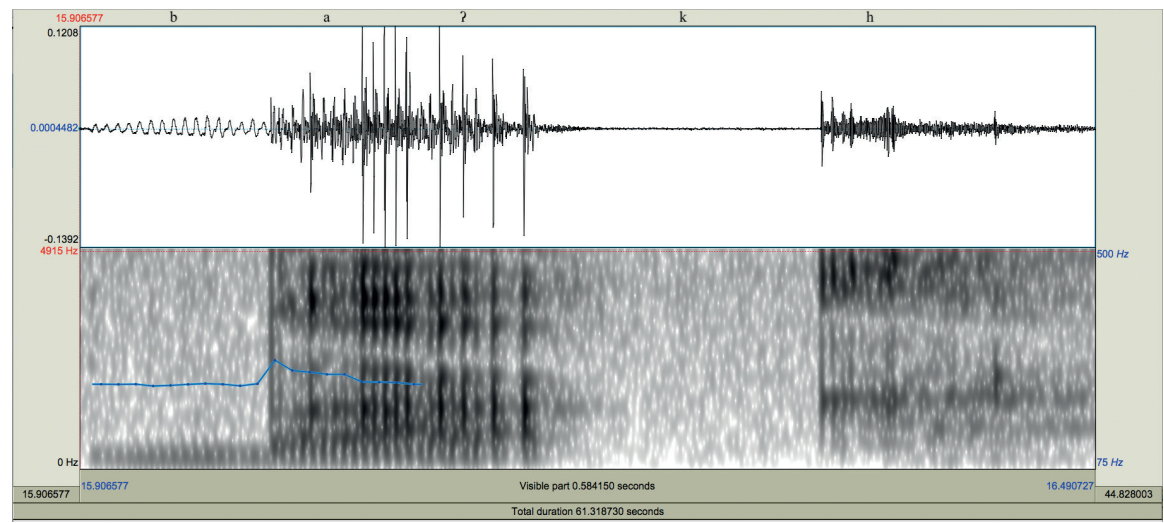

Figura 5. Oscilograma y espectrograma de la palabra bak [bá?k $\left.{ }^{\mathrm{h}}\right]$ 'espalda'

La Figura 5 muestra la realización de la palabra bak [bá?k $\left.{ }^{\mathrm{h}}\right]$ 'espalda'. En este caso, igualmente aparecen pulsaciones irregulares y no periódicas después de la vocal corta [a] antes de la oclusiva velar sorda [k]. Estas pulsaciones son incluso más notables para esta palabra que las que ocurren en el vocablo bit 'trozo', como se nota tanto en el oscilograma como en el espectrograma. La línea azul define el límite de la vocal corta.

En contraste con esto, cuando en una palabra la vocal corta va seguida de una consonante oclusiva sonora /b, d, g/ en posición final de palabra, nunca aparecen las pulsaciones glotales propias de la voz quebrada. La Figura 6 presenta la realización de la palabra dag [dág] 'perseguir'. 


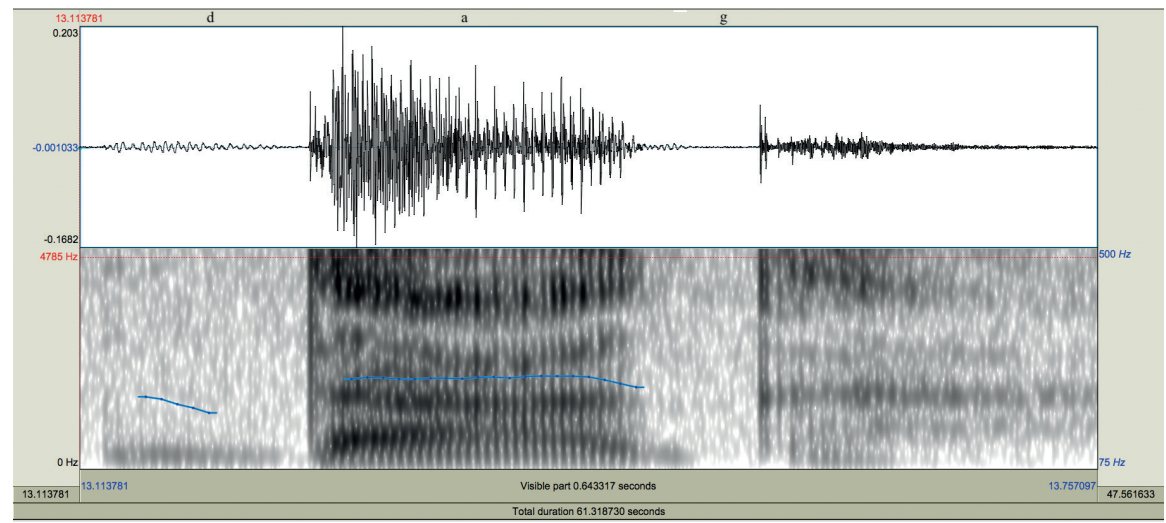

Figura 6. Oscilograma y espectrograma de la palabra dag [dág] 'perseguir'

Las pulsaciones de la onda de sonido correspondientes a la vocal son totalmente regulares hasta el mismo inicio de la oclusión velar. En este respecto, es interesante mencionar que las consonantes oclusivas sonoras /b, d, g/ son pronunciadas, en realidad, de manera ensordecida, como se puede ver en el espectrograma de la Figura 6 para el caso de la consonante $/ \mathrm{g} /$.

Asimismo, tampoco aparecen pulsaciones glotales irregulares en la vocal antes de las consonantes oclusivas sordas /p, t, k/ si estas no se encuentran en posición final de palabra. Por ejemplo, la Figura 7 presenta la realización de la palabra maká [maká] 'espina', en cuyo caso, al encontrarse la oclusiva sorda seguida de una vocal, no hay trazas de voz quebrada.

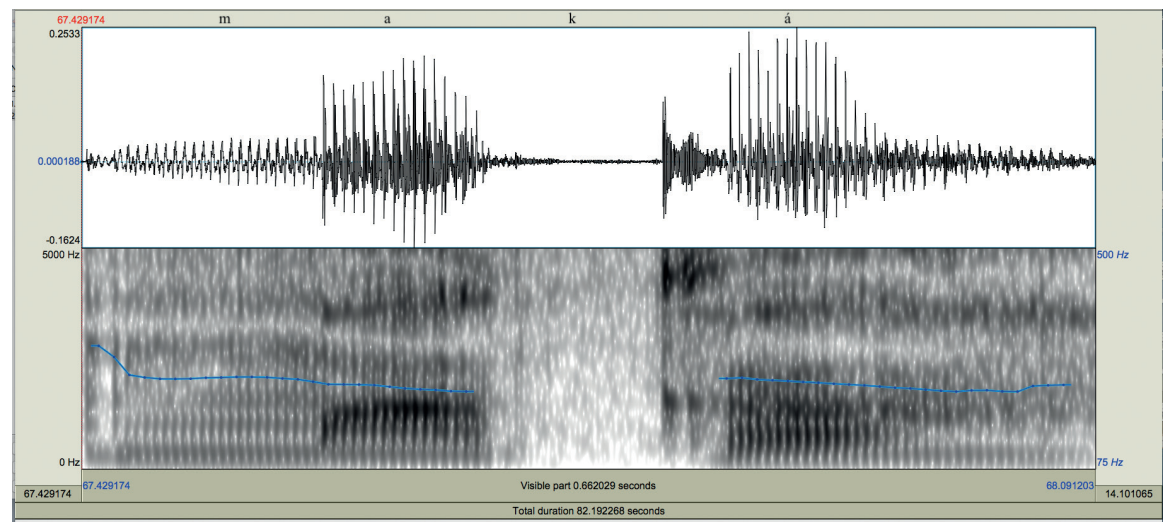

Figura 7. Oscilograma y espectrograma de la palabra maká [maká] 'espina'

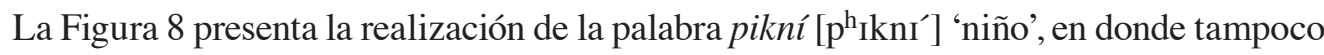
aparecen pulsaciones glotales irregulares, al ir seguida la consonante oclusiva de otra consonante. 


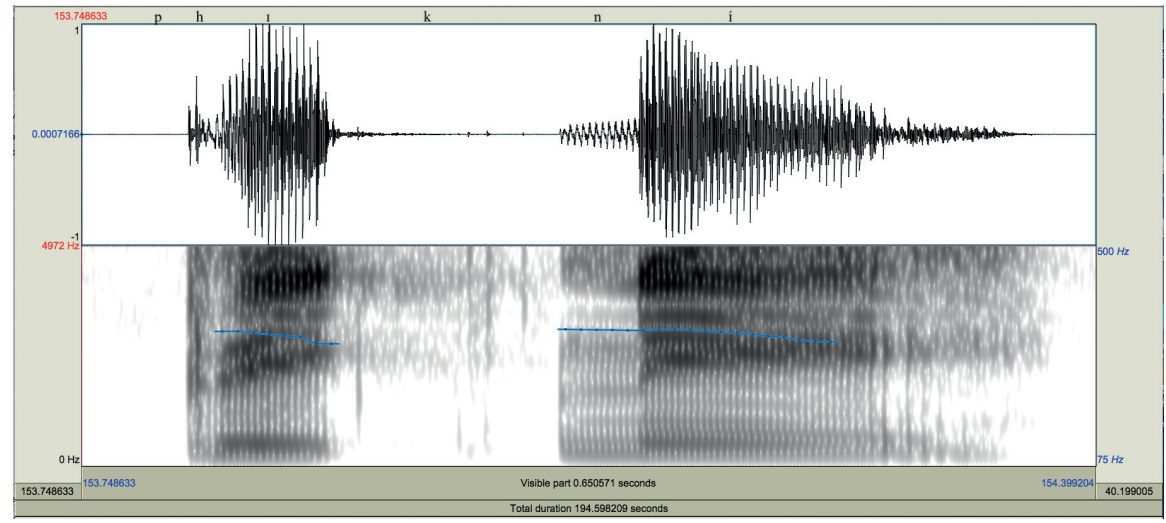

Figura 8. Oscilograma y espectrograma de la palabra pikní [p p $\left.^{\mathrm{h}} \mathrm{kn} \mathrm{I}^{\prime}\right]$ 'muchacho'

Cuando una vocal larga precede a las oclusivas sordas /p, t, k/ normalmente no aparece ninguna huella de voz quebrada como sucede con el vocablo biit [bi 'it ${ }^{\mathrm{h}}$ ] 'golpear', según se ha mostrado en la Figura 3. Sin embargo, a veces también pueden ocurrir pulsaciones glotales irregulares después de este tipo de vocal, como se puede apreciar en la Figura 9 para el caso de la palabra baak [báa?k h] 'ladrar', como fue señalado por Portilla (1993). A pesar de esto, estas pulsaciones no son tan irregulares y no periódicas como las que aparecen en el vocablo bak [bá?k $\left.{ }^{\mathrm{h}}\right]$ 'espalda' (Figura 5).

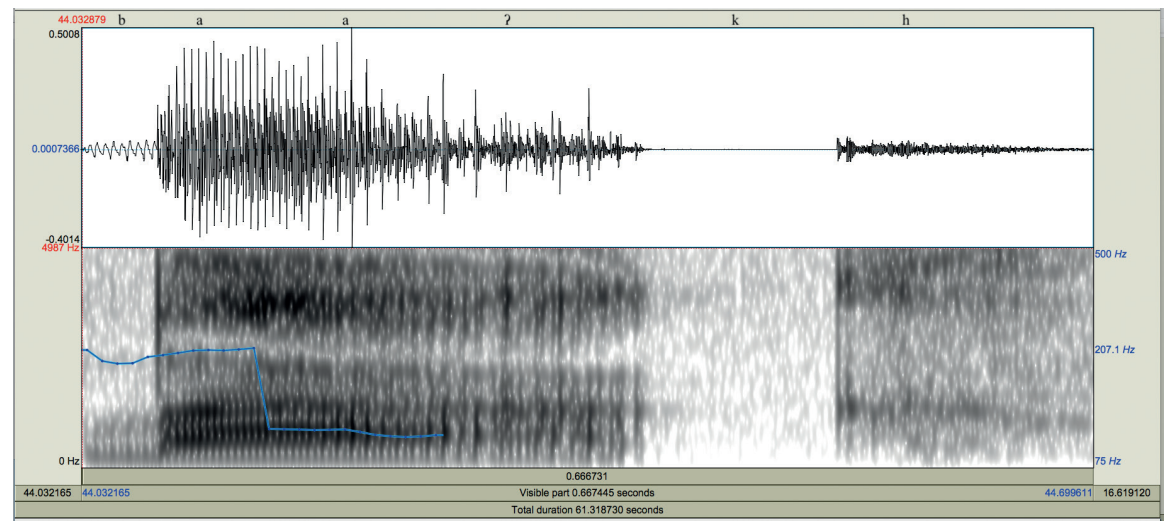

Figura 9. Oscilograma y espectrograma de la palabra baak [báa?k $]$ 'ladrar'

\section{Realización de las oclusivas glotales ante vocales}

En palabras aisladas, según la descripción de Portilla (1993), siempre ocurre una constricción glotal en posición inicial antes de cualquier vocal.

La Figura 10 muestra la realización del vocablo án [?án] 'mano’. En este caso, aparece una oclusión glotal [?], expresada por la ausencia total de vibración de la voz, seguida de una cierta laringalización de la vocal que precede. Este fenómeno puede ser más o menos acusado de manera aleatoria. La Figura 11 presenta la realización de la palabra ánimal [?ánımal] 'animal', en donde se puede apreciar también dicho fenómeno. 


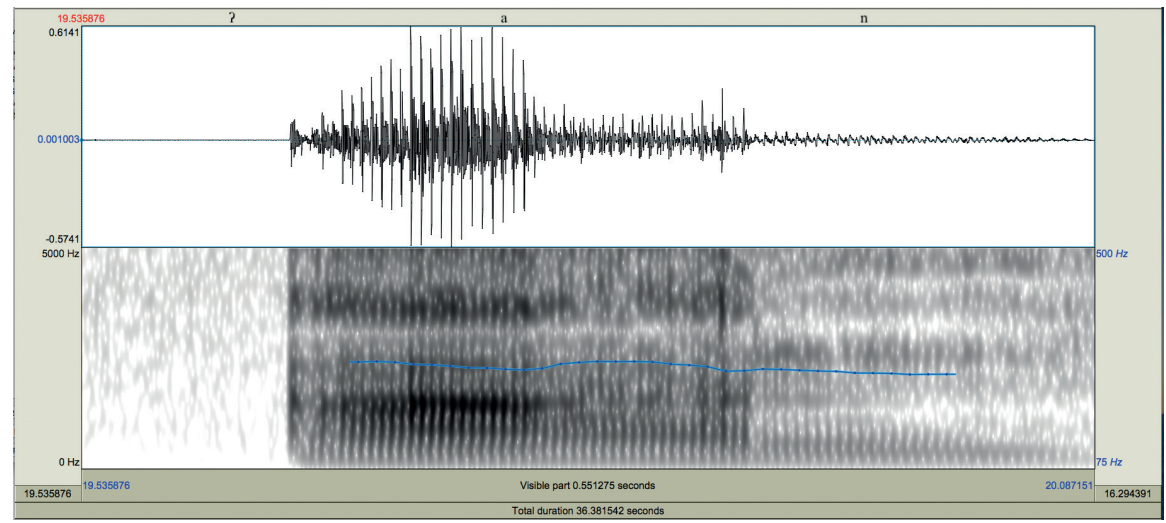

Figura 10. Oscilograma y espectrograma de la palabra an [?án] 'mano'

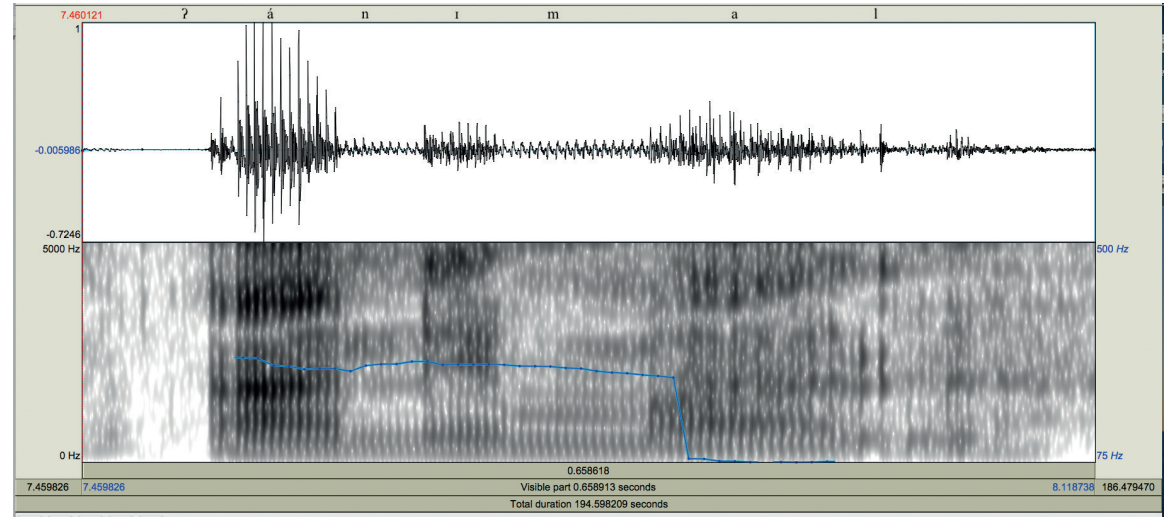

Figura 11. Oscilograma y espectrograma de la palabra ánimal [?ánımal] 'animal'

De manera facultativa, especialmente por énfasis, la constricción glotal también puede corresponder a una consonante fricativa glotal [h]. La Figura 12 muestra la realización del término évi [hévi] 'pesado' con la fricativa glotal. En contraste, la Figura 13 presenta el mismo vocablo pero realizado con una oclusión glotal évi [?évi] 'pesado'.

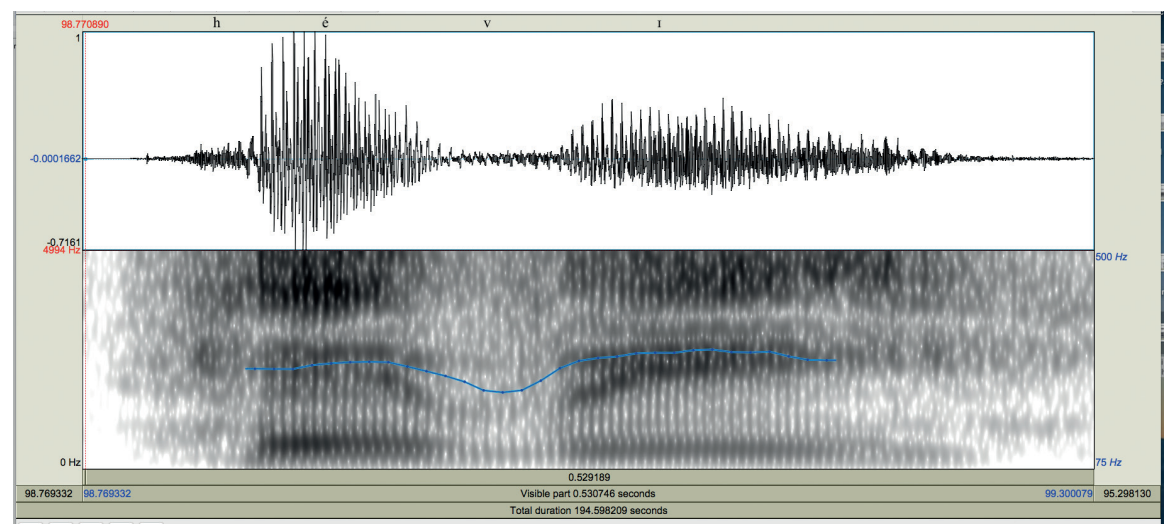

Figura 12. Oscilograma y espectrograma de la palabra évi [hévi] 'pesado' 


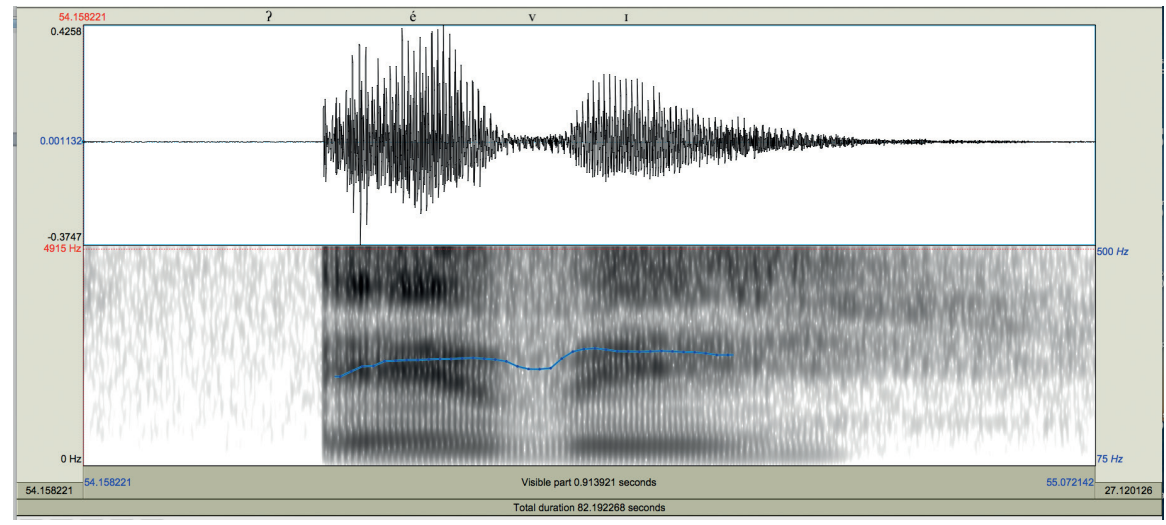

Figura 13. Oscilograma y espectrograma de la palabra évi [?évi] 'pesado'

En oraciones, la oclusión glotal entre vocales es expresada como una interrupción de la onda sonora. La Figura 14 presenta la realización de la frase Aal di ánimal dem [?áal di Pánımal dem] 'Todos los animales'.

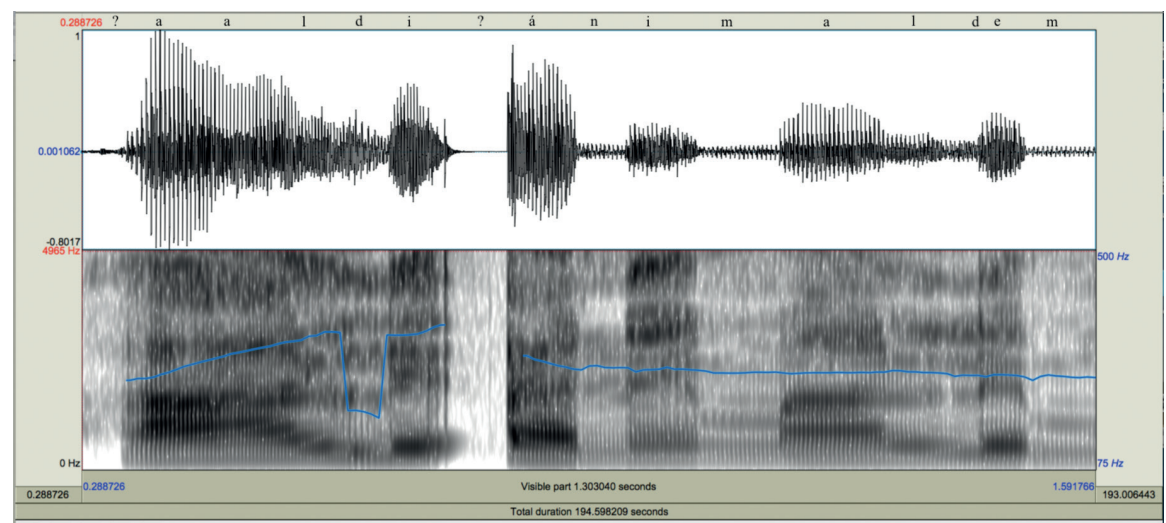

Figura 14. Oscilograma y espectrograma de la frase Áal di ánimal dem [?áal dı ?ánımal dem] 'Todos los animales'

En otros casos, al igual que sucede con las palabras aisladas, la transición entre dos vocales de términos diferentes se da por medio de una consonante fricativa glotal [h], como

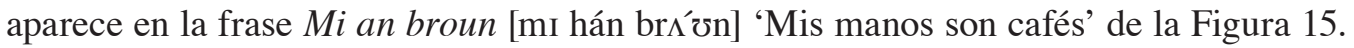




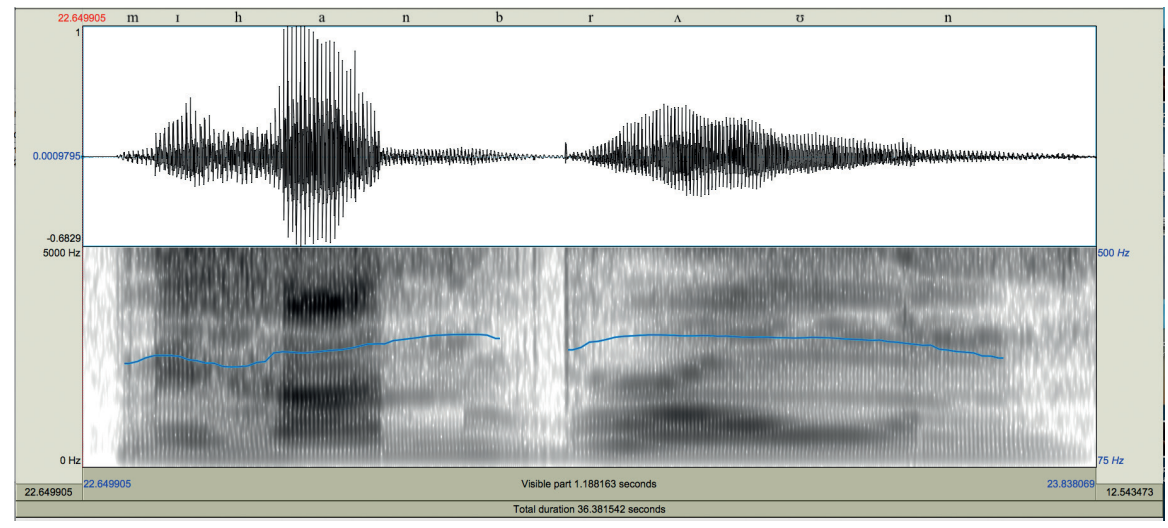

Figura 15. Oscilograma y espectrograma de la frase Mi an broun [mi hán brı' $\mho n]$ 'Mis manos son cafés'

\section{Conclusiones}

Los oscilogramas y los espectrogramas muestran la existencia de perturbaciones glotales en las vocales ante las consonantes oclusivas sordas /p, t, k/ cuando estas se hallan en posición final absoluta. Esta laringalización o voz quebrada es el correlato fonético fundamental de la oclusiva glotal descrita por Portilla (1993). Tal laringalización ocurre, especialmente, cuando las vocales son de longitud corta y, facultativa, cuando estas son largas. Cuando las vocales preceden consonantes oclusivas sonoras en posición final absoluta y cuando las oclusivas sordas se hallan seguidas de una vocal o de otra consonante no aparecen trazas de laringalización en ellas.

También se logró constatar que las vocales en posición inicial absoluta siempre van precedidas o bien de una oclusiva glotal [?] o bien de una fricativa glotal [h], tal como fue descrito por Portilla (1993). Del mismo modo, estos dos segmentos aparecen entre vocales de palabras distintas dentro de las frases.

El origen de las glotales en el inglés criollo limonense se halla, sin duda alguna, en el mismo idioma inglés. Aunque no aparezca una oclusiva glotal ante las consonantes oclusivas en todas las variedades de esta lengua, sí se da su aparición en algunas de ellas. Por ejemplo, en su descripción del inglés hablado, que se basa principalmente en el inglés británico, Brown (1977, p. 29) menciona que, cuando se hallan en posición final, las consonantes oclusivas sordas y /t $\mathrm{f} /$ van precedidas "by a comparatively short vowel and glottalization"4. Y señala: "I include within the term glottalization both tenseness of the vocal cords, which may rise to creakiness in the vowel, and closure of the glottis which forms a glottal stop" .

Sin embargo, hay que mencionar que, según este autor, dicha glotalización aparece no solo después de una vocal (v.gr. leap [li?p]), sino también tras una consonante lateral (v.gr. help [hel?p]) o una nasal (v.gr. sent [sen?t]).

4 "por una vocal comparativamente corta y una glotalización” (Brown, 1977, p. 29, traducción propia).

5 "Incluyo dentro del término glotalización tanto la tensión de las cuerdas vocales, que puede aumentar la voz quebrada en la vocal, como el cierre de la glotis, que forma la oclusiva glotal” (Brown, 1977, p. 29, traducción propia). 
Del mismo modo, Wells (1982, p. 260) señala que /p, t, k, t f/ "are in England often preceded in certain syllable-final enviroments by a glotal stop [?]"6. También menciona que esta glotalización no está tampoco generalizada en la comunidad de los hablantes, aunque es ciertamente común en la variedad de Londres, y añade: "Either this is a new, twentieth-century, phenomenon, or else no phonetician had previously noticed it"7.

El hecho de que esta glotalización ocurra también en el inglés criollo limonense, el cual es un descendiente directo del inglés criollo de Jamaica (veáse Portilla, 1997), apuntaría más bien a que se trata de un rasgo mucho más antiguo en inglés ${ }^{8}$.

En esta línea, es interesante mencionar que Kortlandt (1993, p. 11) supone que esta glotalización asociada a las oclusivas del inglés es un resabio de una serie de consonantes oclusivas preglotalizadas del protogermánico, las cuales también pueden ser detectadas en el escandinavo y en el alto alemán antiguo.

Del mismo modo, la existencia de una oclusión glotal delante de una vocal en posición inicial y en medio de dos vocales ha sido constatado para el inglés estándar, aunque no exactamente en las mismas condiciones descritas para el inglés criollo limonense en el segundo caso (véase Cleghorn y Rugg, 2011). Estos fenómenos también ocurren profusamente en otras lenguas germánicas, como en el alto alemán (véase Pompino-Marshall y Zygis, 2010; Kohler, 1994; inter alia).

La aparición facultativa de una fricativa glotal [h] delante una vocal en posición inicial en lugar de la oclusión glotal en el inglés criollo limonense también tiene su correlato en algunas otras variedades de inglés. Wells (1982, p. 253 y ss.) describe esta misma alternancia, que en términos diacrónicos se relaciona con la desfonologización del segmento /h/ en palabras como hedge 'seto', la cual llega a pronunciarse de la misma forma que edge 'borde': [hedze $\varepsilon d z e \sim$ ? $\varepsilon d z e]$. Según este autor, la desaparición del fonema /h/ prevalece "in the working-class accents of the most of England"9 , en contraste con el estándar. Así mismo, en el inglés criollo de Jamaica no existe el fonema /h/ (Wells, 1982, p. 568 y ss.).

\section{Bibliografía}

Bernard, E. (1969). Phonological study of the Costa Rican Creole English. (Tesis de Maestría). Universidad de Cardiff.

Brown, G. (1977). Listening to spoken English. Londres y Nueva York: Longman.

Cleghorn, T. L. y Rugg, N. M. (2011). Comprehensive articulatory phonetics. CreateSpace Independent Publishing Platform.

Dobson, E. (1968). English pronunciation 1500-1700. Oxford: Clarendon Press.

$6 \quad$ "en Inglaterra, a menudo se encuentran precedidos de una oclusiva glotal [?] en ciertos entornos del final de sílaba" (Wells, 1982, p. 260, traducción propia).

7 "O bien este es un fenómeno nuevo, del siglo XX, o bien ningún fonetista lo había notado previamente" (Wells, 1982, p. 260, traducción propia).

8 El inglés criollo limonense presenta algunos rasgos fonológicos arcaizantes, como la palatalización de /k/ y /g/ ante la vocal anterior /æ/ del inglés moderno temprano (Dobson, 1968, p. 379), por ejemplo, en los vocablos kyat/kját/ 'gato' y gyálik/gjálık/ 'ajo', entre otros.

9 "en los acentos de la clase obrera en la mayor parte de Inglaterra” (Wells, 1982, p. 568, traducción propia). 
Herzfeld, A. (1978). Tense and aspect in Limon Creole: A sociolinguistic view towards a creole continuum. (Tesis doctoral). Universidad de Kansas.

Herzfeld, A. (2002). Mekaytelyuw: La lengua criolla. San José: Editorial de la Universidad de Costa Rica.

Kalita, S., Lalhminghlui, W., Horo, L., Sarmah, P., Prasanna, S., y Dandapat, S. (2017). Acoustic characterization of word-final glottal stop in Mizo and Assam Sora. Interspeech, 10391043. doi: 10.21437/Interspeech.2017-604

Kohler, K. J. (1994). Glottal stops and glottalization in German. Phonetika, 51, 38-51.

Kortlandt, F. (1993). General linguistics and Indo-european reconstruction. [pdf]. Recuperado de https://www.kortlandt.nl/publications/art130e.pdf

Pompino-Marshall, B. y Zygis, M. (2010). Glottal marking of vowel-initial words in German. ZAS Papers in Linguistics, 52, 1-17. Recuperado de https://zaspil.leibniz-zas.de/article/ view/380/381

Portilla, M. (1993). Fonemas segmentales en el criollo inglés de Limón. Revista de Filología y Lingüística de la Universidad de Costa Rica, 19(2), 89-97.

Portilla, M. (1997). Correspondencias tonales y el origen de los criollos ingleses del Atlántico. Revista de Filología y Lingüística de la Universidad de Costa Rica, 23(1), 205-223.

Portilla, M. (2010). Análisis acústico de la longitud vocálica en el inglés criollo de Limón. Revista de Filología y Lingüística de la Universidad de Costa Rica, 36(2), 175-192.

Wells, J. C. (1982). Accents of English. (Vol. 1, 2 y 3). Cambridge: Cambridge University Press.

Wolfe, T. (1970). An exploratory study of morphology and syntax of the English of the Province of Limon, Costa Rica. (Tesis de Licenciatura). Universidad de Costa Rica.

Wright, F. (1974). Limon Creole: A syntactic analysis. (Tesis de Licenciatura). Universidad de Costa Rica.

Wright, F. (1975). Un análisis sintáctico del habla criolla de Limón. Revista de Filología y Lingüística de la Universidad de Costa Rica, 1(2), 149-168. 